\title{
Some Bessel type additive inequalities in inner product spaces
}

\author{
Silvestru Sever Dragomir
}

\begin{abstract}
In this paper we obtain some additive inequalities related to the celebrated Bessel's inequality in inner product spaces. They complement the results obtained by Boas-Bellman, Bombieri, Selberg and Heilbronn, which have been applied for almost orthogonal series and in Number Theory.
\end{abstract}

Mathematics Subject Classification (2010): 46C05, 26D15.

Keywords: Inner product spaces, Bessel's inequality, Schwarz's inequality.

\section{Introduction}

Let $(H ;\langle\cdot, \cdot\rangle)$ be an inner product space over the real or complex number field $\mathbb{K}$. If $\left(e_{i}\right)_{1 \leq i \leq n}$ are orthonormal vectors in the inner product space $H$, i.e., $\left\langle e_{i}, e_{j}\right\rangle=\delta_{i j}$ for all $i, j \in\{1, \ldots, n\}$ where $\delta_{i j}$ is the Kronecker delta, then the following inequality is well known in the literature as Bessel's inequality:

$$
\sum_{i=1}^{n}\left|\left\langle x, e_{i}\right\rangle\right|^{2} \leq\|x\|^{2} \text { for any } x \in H .
$$

For other results related to Bessel's inequality, see [8] - [11] and Chapter XV in the book [14].

In 1941, R. P. Boas [2] and in 1944, independently, R. Bellman [1] proved the following generalization of Bessel's inequality (see also [14, p. 392]):

Theorem 1.1. If $x, y_{1}, \ldots, y_{n}$ are elements of an inner product space $(H ;\langle\cdot, \cdot\rangle)$, then the following inequality holds

$$
\sum_{i=1}^{n}\left|\left\langle x, y_{i}\right\rangle\right|^{2} \leq\|x\|^{2}\left[\max _{1 \leq i \leq n}\left\|y_{i}\right\|^{2}+\left(\sum_{1 \leq i \neq j \leq n}\left|\left\langle y_{i}, y_{j}\right\rangle\right|^{2}\right)^{\frac{1}{2}}\right] .
$$


It is obvious that (1.2) will give for orthonormal families the well known Bessel inequality.

In [7] we pointed out the following Boas-Bellman type inequalities:

$$
\sum_{i=1}^{n}\left|\left\langle x, y_{i}\right\rangle\right|^{2} \leq\|x\| \max _{1 \leq i \leq n}\left|\left\langle x, y_{i}\right\rangle\right|\left\{\sum_{i=1}^{n}\left\|y_{i}\right\|^{2}+\sum_{1 \leq i \neq j \leq n}\left|\left\langle y_{i}, y_{j}\right\rangle\right|\right\}^{\frac{1}{2}}
$$

for any $x, y_{1}, \ldots, y_{n}$ vectors in the inner product space $(H ;\langle\cdot, \cdot\rangle)$.

We also have, see [7]

$$
\begin{aligned}
\sum_{i=1}^{n}\left|\left\langle x, y_{i}\right\rangle\right|^{2} & \leq\|x\|\left(\sum_{i=1}^{n}\left|\left\langle x, y_{i}\right\rangle\right|^{2 p}\right)^{\frac{1}{2 p}} \\
& \times\left\{\left(\sum_{i=1}^{n}\left\|y_{i}\right\|^{2 q}\right)^{\frac{1}{q}}+(n-1)^{\frac{1}{p}}\left(\sum_{1 \leq i \neq j \leq n}\left|\left\langle y_{i}, y_{j}\right\rangle\right|^{q}\right)^{\frac{1}{q}}\right\}^{\frac{1}{2}},
\end{aligned}
$$

for any $x, y_{1}, \ldots, y_{n} \in H, p>1, \frac{1}{p}+\frac{1}{q}=1$.

Further, we recall [7] that

$$
\sum_{i=1}^{n}\left|\left\langle x, y_{i}\right\rangle\right|^{2} \leq\|x\|^{2}\left\{\max _{1 \leq i \leq n}\left\|y_{i}\right\|^{2}+(n-1) \max _{1 \leq i \neq j \leq n}\left|\left\langle y_{i}, y_{j}\right\rangle\right|\right\},
$$

for any $x, y_{1}, \ldots, y_{n} \in H$. It is obvious that (1.5) will give for orthonormal families the well known Bessel inequality.

In 1971, E. Bombieri [3] gave the following generalization of Bessel's inequality.

Theorem 1.2. If $x, y_{1}, \ldots, y_{n}$ are vectors in the inner product space $(H ;(\cdot, \cdot))$, then the following inequality holds:

$$
\sum_{i=1}^{n}\left|\left\langle x, y_{i}\right\rangle\right|^{2} \leq\|x\|^{2} \max _{1 \leq i \leq n}\left\{\sum_{j=1}^{n}\left|\left\langle y_{i}, y_{j}\right\rangle\right|\right\}
$$

It is obvious that if $\left(y_{i}\right)_{1 \leq i \leq n}$ are orthonormal, then from (1.6) one can deduce Bessel's inequality.

It is not widely known, but it appears in a number of places that, the importance of extensions of the Bombieri and Bessel inequality were first shown by J. Sándor (at a Symposium on Mathematical Inequalities, Sibiu, December, 1984), who proved some generalizations of these inequalities, and who was deeply interested in applications in Number Theory. Also, Bessel's inequality and Gram's inequality have been studied by the author and J. Sándor in [12] as well.

Another generalization of Bessel's inequality was obtained by A. Selberg (see for example [14, p. 394]): 
Theorem 1.3. Let $x, y_{1}, \ldots, y_{n}$ be vectors in $H$ with $y_{i} \neq 0 \quad(i=1, \ldots, n)$. Then one has the inequality:

$$
\sum_{i=1}^{n} \frac{\left|\left\langle x, y_{i}\right\rangle\right|^{2}}{\sum_{j=1}^{n}\left|\left\langle y_{i}, y_{j}\right\rangle\right|} \leq\|x\|^{2} .
$$

Another type of inequality related to Bessel's result, was discovered in 1958 by H. Heilbronn [13] (see also [14, p. 395]).

Theorem 1.4. With the assumptions in Theorem 1.2, one has

$$
\sum_{i=1}^{n}\left|\left\langle x, y_{i}\right\rangle\right| \leq\|x\|\left(\sum_{i, j=1}^{n}\left|\left\langle y_{i}, y_{j}\right\rangle\right|\right)^{\frac{1}{2}} \text {. }
$$

In [8] we obtained the following Bombieri type inequalities

$$
\begin{gathered}
\sum_{i=1}^{n}\left|\left\langle x, y_{i}\right\rangle\right|^{2} \leq\|x\| \max _{1 \leq i \leq n}\left|\left\langle x, y_{i}\right\rangle\right|\left(\sum_{i, j=1}^{n}\left|\left\langle y_{i}, y_{j}\right\rangle\right|\right)^{\frac{1}{2}}, \\
\sum_{i=1}^{n}\left|\left\langle x, y_{i}\right\rangle\right|^{2} \\
\leq\|x\| \max _{1 \leq i \leq n}\left|\left\langle x, y_{i}\right\rangle\right|^{\frac{1}{2}}\left(\sum_{i=1}^{n}\left|\left\langle x, y_{i}\right\rangle\right|^{r}\right)^{\frac{1}{2 r}}\left[\sum_{i=1}^{n}\left(\sum_{j=1}^{n}\left|\left\langle y_{i}, y_{j}\right\rangle\right|\right)^{s}\right]^{\frac{1}{2 s}},
\end{gathered}
$$

where $\frac{1}{r}+\frac{1}{s}=1, s>1$,

$$
\begin{aligned}
& \sum_{i=1}^{n}\left|\left\langle x, y_{i}\right\rangle\right|^{2} \\
& \leq\|x\| \max _{1 \leq i \leq n}\left|\left\langle x, y_{i}\right\rangle\right|^{\frac{1}{2}}\left(\sum_{i=1}^{n}\left|\left\langle x, y_{i}\right\rangle\right|\right)^{\frac{1}{2}}\left[\max _{1 \leq i \leq n}\left(\sum_{j=1}^{n}\left|\left\langle y_{i}, y_{j}\right\rangle\right|\right)\right], \\
& \sum_{i=1}^{n}\left|\left\langle x, y_{i}\right\rangle\right|^{2} \\
& \leq\|x\| \max _{1 \leq i \leq n}\left|\left\langle x, y_{i}\right\rangle\right|^{\frac{1}{2}}\left(\sum_{i=1}^{n}\left|\left\langle x, y_{i}\right\rangle\right|^{p}\right)^{\frac{1}{2 p}}\left[\sum_{i=1}^{n}\left(\sum_{j=1}^{n}\left|\left\langle y_{i}, y_{j}\right\rangle\right|^{q}\right)^{\frac{1}{q}}\right]^{\frac{1}{2}},
\end{aligned}
$$

where $p>1, \frac{1}{p}+\frac{1}{q}=1$ and

$$
\sum_{i=1}^{n}\left|\left\langle x, y_{i}\right\rangle\right|^{2} \leq\|x\|^{2}\left\{\sum_{i, j=1}^{n}\left|\left\langle y_{i}, y_{j}\right\rangle\right|^{2}\right\}^{\frac{1}{2}}
$$


for any $x \in H$.

It has been shown that for different selection of vectors the upper bound provided by the inequality (1.13) is some time better other times worse than the one obtained by Bombieri above in (1.6).

In this paper we obtain some inequalities related to the celebrated Bessel's inequality in inner product spaces. They complement the results obtained by BoasBellman, Bombieri, Selberg and Heilbronn above, which have been applied for almost orthogonal series and in Number Theory.

\section{Some results via CBS inequality}

We have:

Theorem 2.1. Let $x, y_{1}, \ldots, y_{n} \in H$ and $\alpha_{1}, \ldots, \alpha_{n} \in \mathbb{C}$. Then

$$
\operatorname{Re}\left(\sum_{j=1}^{n} \alpha_{j}\left\langle y_{j}, x\right\rangle\right) \leq \frac{1}{2}\left[\|x\|^{2}+\sum_{k=1}^{n}\left|\alpha_{k}\right|^{2}\left(\sum_{j, k=1}^{n}\left|\left\langle y_{j}, y_{k}\right\rangle\right|^{2}\right)^{1 / 2}\right]
$$

Proof. We have for any $x, y_{1}, \ldots, y_{n} \in H$ and $\alpha_{1}, \ldots, \alpha_{n} \in \mathbb{C}$ that

$$
\begin{aligned}
0 & \leq\left\|\sum_{j=1}^{n} \alpha_{j} y_{j}-x\right\|^{2}=\left\|\sum_{j=1}^{n} \alpha_{j} y_{j}\right\|^{2}-2 \operatorname{Re}\left\langle\sum_{j=1}^{n} \alpha_{j} y_{j}, x\right\rangle+\|x\|^{2} \\
& =\left\langle\sum_{j=1}^{n} \alpha_{j} y_{j}, \sum_{k=1}^{n} \alpha_{k} y_{k}\right\rangle-2 \operatorname{Re}\left(\sum_{j=1}^{n} \alpha_{j}\left\langle y_{j}, x\right\rangle\right)+\|x\|^{2} \\
& =\sum_{j, k=1}^{n} \alpha_{j} \overline{\alpha_{k}}\left\langle y_{j}, y_{k}\right\rangle-2 \operatorname{Re}\left(\sum_{j=1}^{n} \alpha_{j}\left\langle y_{j}, x\right\rangle\right)+\|x\|^{2},
\end{aligned}
$$

which implies the inequality

$$
\operatorname{Re}\left(\sum_{j=1}^{n} \alpha_{j}\left\langle y_{j}, x\right\rangle\right) \leq \frac{1}{2}\left[\|x\|^{2}+\sum_{j, k=1}^{n} \alpha_{j} \overline{\alpha_{k}}\left\langle y_{j}, y_{k}\right\rangle\right]
$$

for which the term $\sum_{j, k=1}^{n} \alpha_{j} \overline{\alpha_{k}}\left\langle y_{j}, y_{k}\right\rangle$ is obviously nonnegative for any $y_{1}, \ldots, y_{n} \in H$ and $\alpha_{1}, \ldots, \alpha_{n} \in \mathbb{C}$.

By using the Cauchy-Buniakowski-Schwarz's inequality for double sums,

$$
\sum_{j, k=1}^{n}\left|a_{j k} b_{j k}\right| \leq\left(\sum_{j, k=1}^{n}\left|a_{j k}\right|^{2}\right)^{1 / 2}\left(\sum_{j, k=1}^{n}\left|b_{j k}\right|^{2}\right)^{1 / 2}
$$


for complex numbers $a_{j k}, b_{j k}$ where $j, k \in\{1, \ldots, n\}$, then we have

$$
\begin{aligned}
\sum_{j, k=1}^{n} \alpha_{j} \overline{\alpha_{k}}\left\langle y_{j}, y_{k}\right\rangle & =\left|\sum_{j, k=1}^{n} \alpha_{j} \overline{\alpha_{k}}\left\langle y_{j}, y_{k}\right\rangle\right| \leq \sum_{j, k=1}^{n}\left|\alpha_{j} \overline{\alpha_{k}}\right|\left|\left\langle y_{j}, y_{k}\right\rangle\right| \\
& \leq\left(\sum_{j, k=1}^{n}\left|\alpha_{j} \overline{\alpha_{k}}\right|^{2}\right)^{1 / 2}\left(\sum_{j, k=1}^{n}\left|\left\langle y_{j}, y_{k}\right\rangle\right|^{2}\right)^{1 / 2} \\
& =\left(\sum_{j, k=1}^{n}\left|\alpha_{j}\right|^{2}\left|\overline{\alpha_{k}}\right|^{2}\right)^{1 / 2}\left(\sum_{j, k=1}^{n}\left|\left\langle y_{j}, y_{k}\right\rangle\right|^{2}\right)^{1 / 2} \\
& =\left(\sum_{j=1}^{n}\left|\alpha_{j}\right|^{2} \sum_{k=1}^{n}\left|\alpha_{k}\right|^{2}\right)^{1 / 2}\left(\sum_{j, k=1}^{n}\left|\left\langle y_{j}, y_{k}\right\rangle\right|^{2}\right)^{1 / 2} \\
& =\sum_{k=1}^{n}\left|\alpha_{k}\right|^{2}\left(\sum_{j, k=1}^{n}\left|\left\langle y_{j}, y_{k}\right\rangle\right|^{2}\right)^{1 / 2}
\end{aligned}
$$

for any $y_{1}, \ldots, y_{n} \in H$ and $\alpha_{1}, \ldots, \alpha_{n} \in \mathbb{C}$.

By making use of (2.2) and (2.3) we get the desired result (2.1).

Corollary 2.2. With the assumptions of Theorem 2.1 and for $p \geq 1$ we have

$$
\sum_{j=1}^{n}\left|\left\langle x, y_{j}\right\rangle\right|^{p} \leq \frac{1}{2}\left[\|x\|^{2}+\sum_{k=1}^{n}\left|\left\langle x, y_{k}\right\rangle\right|^{2(p-1)}\left(\sum_{j, k=1}^{n}\left|\left\langle y_{j}, y_{k}\right\rangle\right|^{2}\right)^{1 / 2}\right] .
$$

Proof. If we take in (2.1) $\alpha_{j}=\left\langle x, y_{j}\right\rangle\left|\left\langle x, y_{j}\right\rangle\right|^{p-2}$ then we get

$$
\begin{aligned}
& \operatorname{Re}\left(\sum_{j=1}^{n}\left\langle x, y_{j}\right\rangle\left|\left\langle x, y_{j}\right\rangle\right|^{p-2}\left\langle y_{j}, x\right\rangle\right) \\
& \leq \frac{1}{2}\left[\|x\|^{2}+\left.\left.\sum_{k=1}^{n}\left|\left\langle x, y_{j}\right\rangle\right|\left\langle x, y_{j}\right\rangle\right|^{p-2}\right|^{2}\left(\sum_{j, k=1}^{n}\left|\left\langle y_{j}, y_{k}\right\rangle\right|^{2}\right)^{1 / 2}\right],
\end{aligned}
$$

which is equivalent to (2.4).

Remark 2.3. If we take in (2.4) $p=1$, then we get the following Heilbronn type inequality

$$
\sum_{j=1}^{n}\left|\left\langle x, y_{j}\right\rangle\right| \leq \frac{1}{2}\left[\|x\|^{2}+n\left(\sum_{j, k=1}^{n}\left|\left\langle y_{j}, y_{k}\right\rangle\right|^{2}\right)^{1 / 2}\right]
$$

for any $x, y_{1}, \ldots, y_{n} \in H$. 
If we take in (2.4) $p=2$, then we get

$$
\sum_{j=1}^{n}\left|\left\langle x, y_{j}\right\rangle\right|^{2} \leq \frac{1}{2}\left[\|x\|^{2}+\sum_{k=1}^{n}\left|\left\langle x, y_{k}\right\rangle\right|^{2}\left(\sum_{j, k=1}^{n}\left|\left\langle y_{j}, y_{k}\right\rangle\right|^{2}\right)^{1 / 2}\right],
$$

that is equivalent to (see also [10])

$$
\left[2-\left(\sum_{j, k=1}^{n}\left|\left\langle y_{j}, y_{k}\right\rangle\right|^{2}\right)^{1 / 2}\right] \sum_{j=1}^{n}\left|\left\langle x, y_{j}\right\rangle\right|^{2} \leq\|x\|^{2}
$$

for any $x, y_{1}, \ldots, y_{n} \in H$.

The inequality (2.7) is meaningful if

$$
2 \geq\left(\sum_{j, k=1}^{n}\left|\left\langle y_{j}, y_{k}\right\rangle\right|^{2}\right)^{1 / 2}
$$

Also if

$$
1 \geq\left(\sum_{j, k=1}^{n}\left|\left\langle y_{j}, y_{k}\right\rangle\right|^{2}\right)^{1 / 2}
$$

then

$$
\sum_{j=1}^{n}\left|\left\langle x, y_{j}\right\rangle\right|^{2} \leq\left[2-\left(\sum_{j, k=1}^{n}\left|\left\langle y_{j}, y_{k}\right\rangle\right|^{2}\right)^{1 / 2}\right] \sum_{j=1}^{n}\left|\left\langle x, y_{j}\right\rangle\right|^{2} \leq\|x\|^{2},
$$

for any $x \in H$, which improves Bessel's inequality.

We observe that if the family of vectors $\left\{y_{1}, \ldots, y_{n}\right\}$ is orthogonal, then

$$
\sum_{j, k=1}^{n}\left|\left\langle y_{j}, y_{k}\right\rangle\right|^{2}=\sum_{k=1}^{n}\|y\|_{k}^{4}
$$

so, if we assume that

$$
\sum_{k=1}^{n}\|y\|_{k}^{4} \leq 1
$$

then by (2.8) we get the refinement of Bessel's inequality

$$
\sum_{j=1}^{n}\left|\left\langle x, y_{j}\right\rangle\right|^{2} \leq\left[2-\left(\sum_{k=1}^{n}\|y\|_{k}^{4}\right)^{1 / 2}\right] \sum_{j=1}^{n}\left|\left\langle x, y_{j}\right\rangle\right|^{2} \leq\|x\|^{2} .
$$


Corollary 2.4. With the assumptions of Theorem 2.1 we have

$$
\begin{aligned}
& \sum_{j=1}^{n} \frac{\left|\left\langle x, y_{j}\right\rangle\right|^{2}}{\sum_{k=1}^{n}\left|\left\langle y_{k}, y_{j}\right\rangle\right|} \\
& \leq \frac{1}{2}\left[\|x\|^{2}+\sum_{j=1}^{n} \frac{\left|\left\langle x, y_{j}\right\rangle\right|^{2}}{\left(\sum_{k=1}^{n} \mid\left\langle y_{k}, y_{j}\right\rangle\right)^{2}}\left(\sum_{j, k=1}^{n}\left|\left\langle y_{j}, y_{k}\right\rangle\right|^{2}\right)^{1 / 2}\right],
\end{aligned}
$$

for any $x \in H$.

Proof. We take in (2.1)

$$
\alpha_{j}=\frac{\left\langle x, y_{j}\right\rangle}{\sum_{k=1}^{n}\left|\left\langle y_{k}, y_{j}\right\rangle\right|}, j=1, \ldots, n
$$

to get $(2.10)$.

Using the Schwarz's inequality we get from (2.4) that

$$
\begin{aligned}
& \sum_{j=1}^{n}\left|\left\langle x, y_{j}\right\rangle\right|^{p} \\
& \leq \frac{1}{2}\|x\|^{2}\left[1+\|x\|^{2(p-2)} \sum_{k=1}^{n}\left\|y_{k}\right\|^{2(p-1)}\left(\sum_{j, k=1}^{n}\left|\left\langle y_{j}, y_{k}\right\rangle\right|^{2}\right)^{1 / 2}\right],
\end{aligned}
$$

for any $x, y_{1}, \ldots, y_{n} \in H$ and $p \geq 1$.

For $p=2$ we get

$$
\sum_{j=1}^{n}\left|\left\langle x, y_{j}\right\rangle\right|^{2} \leq \frac{1}{2}\|x\|^{2}\left[1+\sum_{k=1}^{n}\left\|y_{k}\right\|^{2}\left(\sum_{j, k=1}^{n}\left|\left\langle y_{j}, y_{k}\right\rangle\right|^{2}\right)^{1 / 2}\right],
$$

for any $x, y_{1}, \ldots, y_{n} \in H$.

From (2.10) we also get Selberg's type inequality

$$
\begin{aligned}
& \sum_{j=1}^{n} \frac{\left|\left\langle x, y_{j}\right\rangle\right|^{2}}{\sum_{k=1}^{n}\left|\left\langle y_{k}, y_{j}\right\rangle\right|} \\
& \leq \frac{1}{2}\|x\|^{2}\left[1+\sum_{j=1}^{n} \frac{\left\|y_{j}\right\|^{2}}{\left(\sum_{k=1}^{n}\left|\left\langle y_{k}, y_{j}\right\rangle\right|\right)^{2}}\left(\sum_{j, k=1}^{n}\left|\left\langle y_{j}, y_{k}\right\rangle\right|^{2}\right)^{1 / 2}\right],
\end{aligned}
$$

for any $x, y_{1}, \ldots, y_{n} \in H$.

Theorem 2.5. Let $x, y_{1}, \ldots, y_{n} \in H$ and $\alpha_{1}, \ldots, \alpha_{n} \in \mathbb{C}$. Then

$$
\operatorname{Re}\left(\sum_{j=1}^{n} \alpha_{j}\left\langle y_{j}, x\right\rangle\right) \leq \frac{1}{2}\left[\|x\|^{2}+\max _{k \in\{1, \ldots, n\}}\left\{\sum_{j=1}^{n}\left|\left\langle y_{j}, y_{k}\right\rangle\right|\right\} \sum_{k=1}^{n}\left|a_{k}\right|^{2}\right]
$$


Proof. By using the Cauchy-Buniakowski-Schwarz's weighted inequality for double sums,

$$
\sum_{j, k=1}^{n} m_{j k}\left|a_{j k} b_{j k}\right| \leq\left(\sum_{j, k=1}^{n} m_{j k}\left|a_{j k}\right|^{2}\right)^{1 / 2}\left(\sum_{j, k=1}^{n} m_{j k}\left|b_{j k}\right|^{2}\right)^{1 / 2}
$$

for complex numbers $a_{j k}, b_{j k}$ and nonnegative numbers $m_{j k}$ where $j, k \in\{1, \ldots, n\}$, then we have

$$
\begin{aligned}
& \sum_{j, k=1}^{n} \alpha_{j} \overline{\alpha_{k}}\left\langle y_{j}, y_{k}\right\rangle \\
& =\left|\sum_{j, k=1}^{n} \alpha_{j} \overline{\alpha_{k}}\left\langle y_{j}, y_{k}\right\rangle\right| \leq \sum_{j, k=1}^{n}\left|\alpha_{j} \overline{\alpha_{k}}\right|\left|\left\langle y_{j}, y_{k}\right\rangle\right|=\sum_{j, k=1}^{n}\left|\alpha_{j}\right|\left|a_{k}\right|\left|\left\langle y_{j}, y_{k}\right\rangle\right| \\
& \leq\left(\sum_{j, k=1}^{n}\left|\left\langle y_{j}, y_{k}\right\rangle\right|\left|a_{j}\right|^{2}\right)^{1 / 2}\left(\sum_{j, k=1}^{n}\left|\left\langle y_{j}, y_{k}\right\rangle\right|\left|a_{k}\right|^{2}\right)^{1 / 2} \\
& =\sum_{j, k=1}^{n}\left|a_{k}\right|^{2}\left|\left\langle y_{j}, y_{k}\right\rangle\right| .
\end{aligned}
$$

Now, observe that

$$
\begin{aligned}
\sum_{j, k=1}^{n}\left|a_{k}\right|^{2}\left|\left\langle y_{j}, y_{k}\right\rangle\right| & =\sum_{k=1}^{n}\left|a_{k}\right|^{2}\left(\sum_{j=1}^{n}\left|\left\langle y_{j}, y_{k}\right\rangle\right|\right) \\
& \leq \max _{k \in\{1, \ldots, n\}}\left\{\sum_{j=1}^{n}\left|\left\langle y_{j}, y_{k}\right\rangle\right|\right\} \sum_{k=1}^{n}\left|a_{k}\right|^{2}
\end{aligned}
$$

which proves the desired inequality (2.14).

Corollary 2.6. With the assumptions of Theorem 2.5 and for $p \geq 1$ we have

$$
\sum_{j=1}^{n}\left|\left\langle x, y_{j}\right\rangle\right|^{p} \leq \frac{1}{2}\left[\|x\|^{2}+\max _{k \in\{1, \ldots, n\}}\left\{\sum_{j=1}^{n}\left|\left\langle y_{j}, y_{k}\right\rangle\right|\right\} \sum_{k=1}^{n}\left|\left\langle x, y_{k}\right\rangle\right|^{2(p-1)}\right] \text {. }
$$

Proof. If we take in (2.14) $\alpha_{j}=\left\langle x, y_{j}\right\rangle\left|\left\langle x, y_{j}\right\rangle\right|^{p-2}$ then we get

$$
\begin{aligned}
& \operatorname{Re}\left(\sum_{j=1}^{n}\left\langle x, y_{j}\right\rangle\left|\left\langle x, y_{j}\right\rangle\right|^{p-2}\left\langle y_{j}, x\right\rangle\right) \\
& \leq \frac{1}{2}\left[\|x\|^{2}+\left.\left.\max _{k \in\{1, \ldots, n\}}\left\{\sum_{j=1}^{n}\left|\left\langle y_{j}, y_{k}\right\rangle\right|\right\} \sum_{k=1}^{n}\left|\left\langle x, y_{j}\right\rangle\right|\left\langle x, y_{j}\right\rangle\right|^{p-2}\right|^{2}\right],
\end{aligned}
$$

which is equivalent to $(2.16)$. 
Remark 2.7. If we take in (2.16) $p=1$, then we get the following Heilbronn type inequality

$$
\sum_{j=1}^{n}\left|\left\langle x, y_{j}\right\rangle\right| \leq \frac{1}{2}\left[\|x\|^{2}+\max _{k \in\{1, \ldots, n\}}\left\{\sum_{j=1}^{n}\left|\left\langle y_{j}, y_{k}\right\rangle\right|\right\}\right]
$$

for any $x, y_{1}, \ldots, y_{n} \in H$.

If we take in (2.16) $p=2$, then we get

$$
\sum_{j=1}^{n}\left|\left\langle x, y_{j}\right\rangle\right|^{2} \leq \frac{1}{2}\left[\|x\|^{2}+\max _{k \in\{1, \ldots, n\}}\left\{\sum_{j=1}^{n}\left|\left\langle y_{j}, y_{k}\right\rangle\right|\right\} \sum_{k=1}^{n}\left|\left\langle x, y_{j}\right\rangle\right|^{2}\right],
$$

which is equivalent to

$$
\left(2-\max _{k \in\{1, \ldots, n\}}\left\{\sum_{j=1}^{n}\left|\left\langle y_{j}, y_{k}\right\rangle\right|\right\}\right) \sum_{j=1}^{n}\left|\left\langle x, y_{j}\right\rangle\right|^{2} \leq\|x\|^{2}
$$

for any $x, y_{1}, \ldots, y_{n} \in H$.

The inequality (2.19) is meaningful if

$$
2 \geq \max _{k \in\{1, \ldots, n\}}\left\{\sum_{j=1}^{n}\left|\left\langle y_{j}, y_{k}\right\rangle\right|\right\}
$$

Also if

$$
1 \geq \max _{k \in\{1, \ldots, n\}}\left\{\sum_{j=1}^{n}\left|\left\langle y_{j}, y_{k}\right\rangle\right|\right\}
$$

then

$$
\sum_{j=1}^{n}\left|\left\langle x, y_{j}\right\rangle\right|^{2} \leq\left[2-\max _{k \in\{1, \ldots, n\}}\left\{\sum_{j=1}^{n}\left|\left\langle y_{j}, y_{k}\right\rangle\right|\right\}\right] \sum_{j=1}^{n}\left|\left\langle x, y_{j}\right\rangle\right|^{2} \leq\|x\|^{2},
$$

for any $x \in H$, which improves Bessel's inequality.

We observe that if the family of vectors $\left\{y_{1}, \ldots, y_{n}\right\}$ is orthogonal, then

$$
\max _{k \in\{1, \ldots, n\}}\left\{\sum_{j=1}^{n}\left|\left\langle y_{j}, y_{k}\right\rangle\right|\right\}=\max _{k \in\{1, \ldots, n\}}\|y\|_{k}^{2},
$$

so, if we assume that $\max _{k \in\{1, \ldots, n\}}\|y\|_{k}^{2} \leq 1$ then by (2.20) we get

$$
\sum_{j=1}^{n}\left|\left\langle x, y_{j}\right\rangle\right|^{2} \leq\left[2-\max _{k \in\{1, \ldots, n\}}\|y\|_{k}^{2}\right] \sum_{j=1}^{n}\left|\left\langle x, y_{j}\right\rangle\right|^{2} \leq\|x\|^{2}
$$

for any $x \in H$. 
Corollary 2.8. With the assumptions of Theorem 2.5 we have

$$
\begin{aligned}
& \sum_{j=1}^{n} \frac{\left|\left\langle x, y_{j}\right\rangle\right|^{2}}{\sum_{k=1}^{n}\left|\left\langle y_{k}, y_{j}\right\rangle\right|} \\
& \leq \frac{1}{2}\left[\|x\|^{2}+\max _{j \in\{1, \ldots, n\}}\left\{\sum_{k=1}^{n}\left|\left\langle y_{j}, y_{k}\right\rangle\right|\right\} \sum_{k=1}^{n} \frac{\left|\left\langle x, y_{k}\right\rangle\right|^{2}}{\left(\sum_{j=1}^{n}\left|\left\langle y_{k}, y_{j}\right\rangle\right|\right)^{2}}\right],
\end{aligned}
$$

for any $x \in H$.

Proof. We take in (2.1)

$$
\alpha_{k}=\frac{\left\langle x, y_{k}\right\rangle}{\sum_{j=1}^{n}\left|\left\langle y_{k}, y_{j}\right\rangle\right|}, k=1, \ldots, n
$$

to get $(2.10)$.

Using the Schwarz's inequality we get from (2.16) that

$$
\begin{aligned}
& \sum_{j=1}^{n}\left|\left\langle x, y_{j}\right\rangle\right|^{p} \\
& \leq \frac{1}{2}\|x\|^{2}\left[1+\|x\|^{2(p-2)} \max _{k \in\{1, \ldots, n\}}\left\{\sum_{j=1}^{n}\left|\left\langle y_{j}, y_{k}\right\rangle\right|\right\} \sum_{k=1}^{n}\left\|y_{k}\right\|^{2(p-1)}\right],
\end{aligned}
$$

for any $x, y_{1}, \ldots, y_{n} \in H$.

If in this inequality we take $p=2$, then we get

$$
\sum_{j=1}^{n}\left|\left\langle x, y_{j}\right\rangle\right|^{2} \leq \frac{1}{2}\|x\|^{2}\left[1+\max _{k \in\{1, \ldots, n\}}\left\{\sum_{j=1}^{n}\left|\left\langle y_{j}, y_{k}\right\rangle\right|\right\} \sum_{k=1}^{n}\left\|y_{k}\right\|^{2}\right]
$$

for any $x, y_{1}, \ldots, y_{n} \in H$.

From (2.22) we also get the Selberg type inequality

$$
\begin{aligned}
& \sum_{j=1}^{n} \frac{\left|\left\langle x, y_{j}\right\rangle\right|^{2}}{\sum_{k=1}^{n}\left|\left\langle y_{k}, y_{j}\right\rangle\right|} \\
& \leq \frac{1}{2}\|x\|^{2}\left[1+\max _{j \in\{1, \ldots, n\}}\left\{\sum_{k=1}^{n}\left|\left\langle y_{j}, y_{k}\right\rangle\right|\right\} \sum_{k=1}^{n} \frac{\left\|y_{k}\right\|^{2}}{\left(\sum_{j=1}^{n}\left|\left\langle y_{k}, y_{j}\right\rangle\right|\right)^{2}}\right],
\end{aligned}
$$

for any $x, y_{1}, \ldots, y_{n} \in H$.

\section{Related inequalities}

We have: 
Theorem 3.1. Let $x, y_{1}, \ldots, y_{n} \in H$ and $\alpha_{1}, \ldots, \alpha_{n} \in \mathbb{C}$. Then

$$
\operatorname{Re}\left(\sum_{j=1}^{n} \alpha_{j}\left\langle y_{j}, x\right\rangle\right) \leq \frac{1}{2}\left[\|x\|^{2}+\max _{j, k \in\{1, \ldots, n\}}\left\{\left|\left\langle y_{j}, y_{k}\right\rangle\right|\right\}\left(\sum_{j=1}^{n}\left|\alpha_{j}\right|\right)^{2}\right]
$$

and

$$
\operatorname{Re}\left(\sum_{j=1}^{n} \alpha_{j}\left\langle y_{j}, x\right\rangle\right) \leq \frac{1}{2}\left[\|x\|^{2}+\max _{k \in\{1, \ldots, n\}}\left\{\left|\alpha_{k}\right|^{2}\right\} \sum_{j, k=1}^{n}\left|\left\langle y_{j}, y_{k}\right\rangle\right|\right]
$$

Proof. From (2.3) we have

$$
\begin{aligned}
\sum_{j, k=1}^{n} \alpha_{j} \overline{\alpha_{k}}\left\langle y_{j}, y_{k}\right\rangle & =\left|\sum_{j, k=1}^{n} \alpha_{j} \overline{\alpha_{k}}\left\langle y_{j}, y_{k}\right\rangle\right| \leq \sum_{j, k=1}^{n}\left|\alpha_{j} \overline{\alpha_{k}}\right|\left|\left\langle y_{j}, y_{k}\right\rangle\right| \\
& \leq \max _{j, k \in\{1, \ldots, n\}}\left\{\left|\left\langle y_{j}, y_{k}\right\rangle\right|\right\} \sum_{j, k=1}^{n}\left|\alpha_{j} \overline{\alpha_{k}}\right| \\
& =\max _{j, k \in\{1, \ldots, n\}}\left\{\left|\left\langle y_{j}, y_{k}\right\rangle\right|\right\} \sum_{j, k=1}^{n}\left|\alpha_{j}\right|\left|\overline{\alpha_{k}}\right| \\
& =\max _{j, k \in\{1, \ldots, n\}}\left\{\left|\left\langle y_{j}, y_{k}\right\rangle\right|\right\}\left(\sum_{j=1}^{n}\left|\alpha_{j}\right|\right)^{2},
\end{aligned}
$$

for any $x, y_{1}, \ldots, y_{n} \in H$ and $\alpha_{1}, \ldots, \alpha_{n} \in \mathbb{C}$, which proves (3.1).

Similarly, we have

$$
\begin{aligned}
\sum_{j, k=1}^{n} \alpha_{j} \overline{\alpha_{k}}\left\langle y_{j}, y_{k}\right\rangle & =\left|\sum_{j, k=1}^{n} \alpha_{j} \overline{\alpha_{k}}\left\langle y_{j}, y_{k}\right\rangle\right| \leq \sum_{j, k=1}^{n}\left|\alpha_{j} \overline{\alpha_{k}}\right|\left|\left\langle y_{j}, y_{k}\right\rangle\right| \\
& \leq \max _{j, k \in\{1, \ldots, n\}}\left\{\left|\alpha_{j} \overline{\alpha_{k}}\right|\right\} \sum_{j, k=1}^{n}\left|\left\langle y_{j}, y_{k}\right\rangle\right| \\
& =\max _{k \in\{1, \ldots, n\}}\left\{\left|\alpha_{k}\right|^{2}\right\} \sum_{j, k=1}^{n}\left|\left\langle y_{j}, y_{k}\right\rangle\right|
\end{aligned}
$$

for any $x, y_{1}, \ldots, y_{n} \in H$ and $\alpha_{1}, \ldots, \alpha_{n} \in \mathbb{C}$, which proves $(3.2)$.

Corollary 3.2. With the assumptions of Theorem 3.1 and for $p \geq 1$ we have

$$
\sum_{j=1}^{n}\left|\left\langle x, y_{j}\right\rangle\right|^{p} \leq \frac{1}{2}\left[\|x\|^{2}+\max _{j, k \in\{1, \ldots, n\}}\left\{\left|\left\langle y_{j}, y_{k}\right\rangle\right|\right\}\left(\sum_{j=1}^{n}\left|\left\langle x, y_{j}\right\rangle\right|^{p-1}\right)^{2}\right]
$$


and

$$
\sum_{j=1}^{n}\left|\left\langle x, y_{j}\right\rangle\right|^{p} \leq \frac{1}{2}\left[\|x\|^{2}+\max _{k \in\{1, \ldots, n\}}\left\{\left|\left\langle x, y_{j}\right\rangle\right|^{2(p-1)}\right\} \sum_{j, k=1}^{n}\left|\left\langle y_{j}, y_{k}\right\rangle\right|\right]
$$

for any $x, y_{1}, \ldots, y_{n} \in H$.

Proof. If we take in (3.1) and (3.2) $\alpha_{j}=\left\langle x, y_{j}\right\rangle\left|\left\langle x, y_{j}\right\rangle\right|^{p-2}$ then we get (3.3) and (3.4).

Remark 3.3. If we take in (3.3) and (3.4) $p=1$, then we get

$$
\sum_{j=1}^{n}\left|\left\langle x, y_{j}\right\rangle\right| \leq \frac{1}{2}\left[\|x\|^{2}+n^{2} \max _{j, k \in\{1, \ldots, n\}}\left\{\left|\left\langle y_{j}, y_{k}\right\rangle\right|\right\}\right]
$$

and

$$
\sum_{j=1}^{n}\left|\left\langle x, y_{j}\right\rangle\right| \leq \frac{1}{2}\left[\|x\|^{2}+\sum_{j, k=1}^{n}\left|\left\langle y_{j}, y_{k}\right\rangle\right|\right]
$$

for any $x, y_{1}, \ldots, y_{n} \in H$.

If we take in (3.3) and (3.4) $p=2$, then we get

$$
\sum_{j=1}^{n}\left|\left\langle x, y_{j}\right\rangle\right|^{2} \leq \frac{1}{2}\left[\|x\|^{2}+\max _{j, k \in\{1, \ldots, n\}}\left\{\left|\left\langle y_{j}, y_{k}\right\rangle\right|\right\}\left(\sum_{j=1}^{n}\left|\left\langle x, y_{j}\right\rangle\right|\right)^{2}\right]
$$

and

$$
\sum_{j=1}^{n}\left|\left\langle x, y_{j}\right\rangle\right|^{2} \leq \frac{1}{2}\left[\|x\|^{2}+\max _{k \in\{1, \ldots, n\}}\left\{\left|\left\langle x, y_{k}\right\rangle\right|^{2}\right\} \sum_{j, k=1}^{n}\left|\left\langle y_{j}, y_{k}\right\rangle\right|\right]
$$

for any $x, y_{1}, \ldots, y_{n} \in H$.

Using Schwarz's inequality we have from (3.3) and (3.4) that

$$
\begin{aligned}
& \sum_{j=1}^{n}\left|\left\langle x, y_{j}\right\rangle\right|^{p} \\
& \leq \frac{1}{2}\|x\|^{2}\left[1+\|x\|^{2(p-2)} \max _{j, k \in\{1, \ldots, n\}}\left\{\left|\left\langle y_{j}, y_{k}\right\rangle\right|\right\}\left(\sum_{j=1}^{n}\left\|y_{j}\right\|^{p-1}\right)^{2}\right]
\end{aligned}
$$

and

$$
\begin{aligned}
& \sum_{j=1}^{n}\left|\left\langle x, y_{j}\right\rangle\right|^{p} \\
& \leq \frac{1}{2}\|x\|^{2}\left[1+\|x\|^{2(p-2)} \max _{k \in\{1, \ldots, n\}}\left\{\left\|y_{k}\right\|^{2(p-1)}\right\} \sum_{j, k=1}^{n}\left|\left\langle y_{j}, y_{k}\right\rangle\right|\right]
\end{aligned}
$$

for any $x, y_{1}, \ldots, y_{n} \in H$. 
For $p=2$ we get

$$
\sum_{j=1}^{n}\left|\left\langle x, y_{j}\right\rangle\right|^{2} \leq \frac{1}{2}\|x\|^{2}\left[1+\max _{j, k \in\{1, \ldots, n\}}\left\{\left|\left\langle y_{j}, y_{k}\right\rangle\right|\right\}\left(\sum_{j=1}^{n}\left\|y_{j}\right\|\right)^{2}\right]
$$

and

$$
\sum_{j=1}^{n}\left|\left\langle x, y_{j}\right\rangle\right|^{2} \leq \frac{1}{2}\|x\|^{2}\left[1+\max _{k \in\{1, \ldots, n\}}\left\{\left\|y_{k}\right\|^{2}\right\} \sum_{j, k=1}^{n}\left|\left\langle y_{j}, y_{k}\right\rangle\right|\right]
$$

for any $x, y_{1}, \ldots, y_{n} \in H$.

We observe that if $y_{1}, \ldots, y_{n} \in H$ are such that

$$
\max _{j, k \in\{1, \ldots, n\}}\left\{\left|\left\langle y_{j}, y_{k}\right\rangle\right|\right\}\left(\sum_{j=1}^{n}\left\|y_{j}\right\|\right)^{2} \leq 1
$$

then (3.1) provides a refinement of Bessel's inequality. Also, if

$$
\max _{k \in\{1, \ldots, n\}}\left\{\left\|y_{k}\right\|^{2}\right\} \sum_{j, k=1}^{n}\left|\left\langle y_{j}, y_{k}\right\rangle\right| \leq 1,
$$

then (3.12) also provides a refinement of Bessel's inequality.

By using Hölder's inequality we can provide other inequalities as follows:

Theorem 3.4. Let $x, y_{1}, \ldots, y_{n} \in H$ and $\alpha_{1}, \ldots, \alpha_{n} \in \mathbb{C}$. Then for $r, q>1$ with $\frac{1}{r}+\frac{1}{q}=1$

$$
\operatorname{Re}\left(\sum_{j=1}^{n} \alpha_{j}\left\langle y_{j}, x\right\rangle\right) \leq \frac{1}{2}\left[\|x\|^{2}+\left(\sum_{j, k=1}^{n}\left|\left\langle y_{j}, y_{k}\right\rangle\right|^{r}\right)^{1 / r}\left(\sum_{j=1}^{n}\left|\alpha_{j}\right|^{q}\right)^{2 / q}\right]
$$

Proof. From (2.3) and Hölder's inequality we have

$$
\begin{aligned}
\sum_{j, k=1}^{n} \alpha_{j} \overline{\alpha_{k}}\left\langle y_{j}, y_{k}\right\rangle & =\left|\sum_{j, k=1}^{n} \alpha_{j} \overline{\alpha_{k}}\left\langle y_{j}, y_{k}\right\rangle\right| \leq \sum_{j, k=1}^{n}\left|\alpha_{j} \overline{\alpha_{k}}\right|\left|\left\langle y_{j}, y_{k}\right\rangle\right| \\
& \leq\left(\sum_{j, k=1}^{n}\left|\left\langle y_{j}, y_{k}\right\rangle\right|^{r}\right)^{1 / r}\left(\sum_{j, k=1}^{n}\left|\alpha_{j} \overline{\alpha_{k}}\right|^{q}\right)^{1 / q} \\
& =\left(\sum_{j, k=1}^{n}\left|\left\langle y_{j}, y_{k}\right\rangle\right|^{r}\right)^{1 / r}\left(\sum_{j, k=1}^{n}\left|\alpha_{j}\right|^{q}\left|\alpha_{k}\right|^{q}\right)^{1 / q} \\
& =\left(\sum_{j, k=1}^{n}\left|\left\langle y_{j}, y_{k}\right\rangle\right|^{r}\right)^{1 / r}\left(\sum_{j=1}^{n}\left|\alpha_{j}\right|^{q}\right)^{2 / q},
\end{aligned}
$$

for any $x, y_{1}, \ldots, y_{n} \in H$ and $\alpha_{1}, \ldots, \alpha_{n} \in \mathbb{C}$, which proves (3.13). 
Corollary 3.5. With the assumptions of Theorem 3.4 and for $p \geq 1$ we have

$$
\sum_{j=1}^{n}\left|\left\langle x, y_{j}\right\rangle\right|^{p} \leq \frac{1}{2}\left[\|x\|^{2}+\left(\sum_{j, k=1}^{n}\left|\left\langle y_{j}, y_{k}\right\rangle\right|^{r}\right)^{1 / r}\left(\sum_{j=1}^{n}\left|\left\langle x, y_{j}\right\rangle\right|^{q(p-1)}\right)^{2 / q}\right]
$$

for any $x, y_{1}, \ldots, y_{n} \in H$. In particular, we have

$$
\sum_{j=1}^{n}\left|\left\langle x, y_{j}\right\rangle\right| \leq \frac{1}{2}\left[\|x\|^{2}+n^{2 / q}\left(\sum_{j, k=1}^{n}\left|\left\langle y_{j}, y_{k}\right\rangle\right|^{r}\right)^{1 / r}\right]
$$

and

$$
\sum_{j=1}^{n}\left|\left\langle x, y_{j}\right\rangle\right|^{2} \leq \frac{1}{2}\left[\|x\|^{2}+\left(\sum_{j, k=1}^{n}\left|\left\langle y_{j}, y_{k}\right\rangle\right|^{r}\right)^{1 / r}\left(\sum_{j=1}^{n}\left|\left\langle x, y_{j}\right\rangle\right|^{2 q}\right)^{2 / q}\right] .
$$

We observe that, by Schwarz's inequality we get for $p \geq 1$

$$
\begin{aligned}
& \sum_{j=1}^{n}\left|\left\langle x, y_{j}\right\rangle\right|^{p} \\
& \leq \frac{1}{2}\|x\|^{2}\left[1+\|x\|^{2(p-2)}\left(\sum_{j, k=1}^{n}\left|\left\langle y_{j}, y_{k}\right\rangle\right|^{r}\right)^{1 / r}\left(\sum_{j=1}^{n}\left\|y_{j}\right\|^{q(p-1)}\right)^{2 / q}\right],
\end{aligned}
$$

for any $x, y_{1}, \ldots, y_{n} \in H$, where $r, q>1$ with $\frac{1}{r}+\frac{1}{q}=1$.

For $p=2$, we get

$$
\sum_{j=1}^{n}\left|\left\langle x, y_{j}\right\rangle\right|^{2} \leq \frac{1}{2}\|x\|^{2}\left[1+\left(\sum_{j, k=1}^{n}\left|\left\langle y_{j}, y_{k}\right\rangle\right|^{r}\right)^{1 / r}\left(\sum_{j=1}^{n}\left\|y_{j}\right\|^{q}\right)^{2 / q}\right],
$$

for any $x, y_{1}, \ldots, y_{n} \in H$, where $r, q>1$ with $\frac{1}{r}+\frac{1}{q}=1$.

We observe that if $y_{1}, \ldots, y_{n} \in H$ are such that

$$
\left(\sum_{j, k=1}^{n}\left|\left\langle y_{j}, y_{k}\right\rangle\right|^{r}\right)^{1 / r}\left(\sum_{j=1}^{n}\left\|y_{j}\right\|^{q}\right)^{2 / q} \leq 1,
$$

where $r, q>1$ with $\frac{1}{r}+\frac{1}{q}=1$, then (3.18) provides a refinement of Bessel's inequality. Acknowledgement. The author would like to thank the anonymous referee for valuable suggestions that have been implemented in the final version of the paper.

\section{References}

[1] Bellman, R., Almost orthogonal series, Bull. Amer. Math. Soc., 50(1944), 517-519.

[2] Boas, R.P., A general moment problem, Amer. J. Math., 63(1941), 361-370.

[3] Bombieri, E., A note on the large sieve, Acta Arith., 18(1971), 401-404. 
[4] Dragomir, S.S., Discrete Inequalities of the Cauchy-Buniakowsky-Schwartz Type, Nova Science Publishers, Inc., Hauppauge, NY, 2004, x+225 pp., ISBN: 1-59454-049-7.

[5] Dragomir, S.S., Advances in Inequalities of the Schwarz, Grüss and Bessel Type in Inner Product Spaces, Nova Science Publishers, Inc., Hauppauge, NY, 2005, viii+249 pp., ISBN: 1-59454-202-3.

[6] Dragomir, S.S., Advances in Inequalities of the Schwarz, Triangle and Heisenberg Type in Inner Product Spaces, Nova Science Publishers, Inc., New York, 2007, xii+243 pp., ISBN: 978-1-59454-903-8; 1-59454-903-6.

[7] Dragomir, S.S., On the Boas-Bellman inequality in inner product spaces, Bull. Austral. Math. Soc., 69(2004), no. 2, 217-225. Preprint RGMIA Res. Rep. Coll., 6(2003), Supplement, Article 14.

[8] Dragomir, S.S., On the Bombieri inequality in inner product spaces, Libertas Math., 25(2005), 13-26. Preprint RGMIA Res. Rep. Coll., 6(2003), No. 3, Article 5.

[9] Dragomir, S.S., Some Bombieri type inequalities in inner product spaces, J. Indones. Math. Soc., 10(2004), no. 2, 91-98. Preprint RGMIA Res. Rep. Coll., 6(2003), Supplement, Article 16.

[10] Dragomir, S.S., Mond, B., On the Boas-Bellman generalisation of Bessel's inequality in inner product spaces, Italian J. of Pure \& Appl. Math., 3(1998), 29-35.

[11] Dragomir, S.S., Mond, B., Pečarić, J.E., Some remarks on Bessel's inequality in inner product spaces, Stud. Univ. Babeş-Bolyai Math., 37(1992), no. 4, 77-86.

[12] Dragomir, S.S., Sándor, J., On Bessels's and Gram's inequalities in prehilbertian spaces, Periodica Math. Hungarica, 29(1994), no. 3, 197-205.

[13] Heilbronn, H., On the averages of some arithmetical functions of two variables, Mathematica, 5(1958), 1-7.

[14] Mitrinović, D.S., Pečarić, J.E., Fink, A.M., Classical and New Inequalities in Analysis, Kluwer Academic Publishers, Dordrecht/Boston/London, 1993.

Silvestru Sever Dragomir

Mathematics, College of Engineering \& Science

Victoria University, PO Box 14428

Melbourne City, MC 8001, Australia

e-mail: sever.dragomir@vu.edu.au 